\title{
Les huîtres gay de Monsieur Marcel
}

\author{
Franc Schuerewegen
}

La scène se passe au Grand Hôtel de Balbec, à l'heure du déjeuner. La marquise de Villeparisis, que Proust dit "très gourmande", ' est venue s'asseoir un instant à la table du narrateur et de sa grand-mère. C'est une politesse qu'elle leur fait, la grande dame sait se montrer aimable. La conversation porte sur la nourriture. Nous lisons ceci:

"Mais il semble que vous ne mangez jamais d'huitres, nous dit Mme de Villeparisis (augmentant l'impression de dégoût que j'avais à cette heure-là, car la chaire vivante des huîtres me répugnait encore plus que la viscosité des méduses ne me ternissait la plage de Balbec); elles sont exquises sur cette côte!" (JF, II, 56)

Évidemment, de gustibus etc. On peut aimer ou ne pas aimer les huîtres, c'est personnel, à chacun sa liberté. Il n'empêche que le héros du livre est bien jeune encore et qu'il en va de la gourmandise comme de la sagesse: il faut la cultiver, très peu est donné au départ.

Or, voici le même personnage quelque cent cinquante pages plus loin, dans la même salle à manger, ayant fini de déjeuner:

Je restais maintenant volontiers à table pendant qu'on desservait [...] je cherchais à retrouver dans la réalité, j'aimais comme quelque chose de poétique, le geste interrompu des couteaux encore de travers, la rondeur bombée d'une serviette [...] la promenade des chaises vieillottes qui deux fois par jour viennent s'installer autour de la nappe, dressée sur la table ainsi que sur un autel où sont célébrées les fêtes de la gourmandise et sur laquelle au fond des huîtres quelques gouttes d'eau lustrale restent comme dans de petits bénitiers de pierre. (JF, II, 224)

I. A l'ombre des jeunes filles en fleurs, A la Recherche du temps perdu, éd. publiée sous la direction de J.-Y. Tadié, "Bibliothèque de la Pléiade", t. II, p. 54. Toutes nos références à Proust concernent cette édition. $\mathrm{s}=\mathrm{Du}$ Côté de chez Swann, $\mathrm{CG}=$ Le Côté de Guermantes, $\mathrm{sG}=$ Sodome et Gomorrhe, $\mathrm{P}=$ La Prisonnière, $\mathrm{TR}=$ Le Temps retrouvé. 
Certes, ce qui compte, c'est l'aspect esthétique, et non pas gastronomique. Proust décrit la coquille, rien n'est dit de la chair. Mais la coquille vient avec la chair, l'une ne va pas sans l'autre, l'une évoque l'autre, c'est bien là le problème. Simple raisonnement de bon sens: s'il reste des écailles sur table, c'est qu'il y eut des huîtres au menu. Chose curieuse: cela ne semble avoir ni gêné, ni offusqué le héros qui n'est pas allé vomir dans un coin, que nous voyons au contraire tout content, tout béat. Proust aurait-il donc commis une inadvertance? Aurait-il oublié l'épisode du premier déjeuner et la parenthèse sur le dégoût? C'est peu probable, croyons-nous. Partons plutôt de l'idée qu'une évolution est suggérée, un passage à un niveau supérieur, un apprentissage, si on veut ( $A$ la recherche $d u$ temps perdu est aussi un Bildungsroman). Gastronomiquement parlant, le héros a progressé, mûri. Il a emporté une petite victoire sur luimême. L'huître était un objet de dégoût pour lui, elle ne l'est plus vraiment ici.

C'est la visite à l'atelier d'Elstir, nous dira-t-on, qui est à l'origine du changement qu'on observe. Rappelons-nous: dans les jours précédant ce second déjeuner, le héros est allé sonner à la porte de la villa où travaille le grand peintre. Les images qu'il a vues l'ont grandement impressionné: elles lui ont appris à regarder les choses d'un autre oeil, en artiste, en esthète. Grâce à Elstir, le héros est devenu sensible à la beauté du quotidien, au charme des "choses usuelles". Mais cette explication, pour séduisante qu' elle puisse paraître, ne nous convainc pas vraiment. C'est que le grand peintre apparaît essentiellement dans le livre comme un éducateur du regard, un "professeur de beauté". ${ }^{3}$ Or, dans le passage qu'on vient de citer, le "beau" et le "bon" ne sont pas séparables. Ce sont "les fêtes de la gourmandise" qu'on célèbre à l'hôtel. L'esthétique et le gastronomique vont main dans la main, Proust ne distingue pas vraiment entre les deux registres. La question demeure donc: que s'est-il passé? D'où vient cette curieuse

2. L'expression apparaît dans l'étude sur "[Chardin et Rembrandt]" où nous voyons un autre jeune homme "écoeuré" par la laideur des choses, jusqu'à ce que l'oeuvre de Chardin lui apprenne à surmonter son dégoût: "Si en regardant un Chardin vous pouvez vous dire: cela est intime, est confortable, est vivant comme une cuisine, en vous promenant dans une cuisine vous vous direz: cela est grand, cela est beau comme un Chardin" (Contre Sainte-Beuve précédé de Pastiches et mélanges et suivi de Essais et articles, éd. établié par P. Clarac avec la coll. d'Y. Sandre, "Bibliothèque de la Pléiade", 197I, p. 374). Notons que dans ce texte, la peinture de Chardin est dite "savoureuse" (p. 376), ce qu'on peut voir comme l'amorce d'une gastronomo-esthétique à venir.

3. "Un professeur de beauté" [sur Montesquiou], Les Arts de la vie, I5 aôut 1905, repris dans Contre Sainte-Beuve, éd. cité, p. so6 et suiv. 
volte-face? Pourquoi l'huître est-elle soudainement devenue inoffensive, voire appétissante, alors qu'elle était répugnante au départ?

Quittons Balbec un instant pour nous rendre à Doncières, ville militaire. Dans une autre salle à manger, bien chauffée, bien confortable, le marquis de SaintLoup dîne avec quelques amis. Le héros a été convié aux agapes. Une fois de plus, nous le voyons tout content, souriant d'aise. C'est que la nourriture est vraiment exquise dans ce restaurant où on sert, entre autres, des huîtres:

Je me sentais séparé $[. .$.$] de toutes les préoccupations extérieures [...] par la$ chaleur de cette petite salle à manger, par la saveur des plats raffinés qu'on nous servait. Ils donnaient autant de plaisir à mon imagination qu'à ma gourmandise; parfois le petit morceau de nature d'où ils avaient été extraits, bénitier rugueux de l'huître dans lequel restent quelques gouttes d'eau salée, ou sarment noueux, pampres jaunis d'une grappe de raisin, les entourait encore, incomestible, poétique et lointain comme un paysage, et faisant se succéder au cours du dîner les évocations d'une sieste sous une vigne et d'une promenade en mer. (CG, II, 4I6)

C'est encore l'écaille (“incomestible”) qui est évoquée et les vertus plastiques qu'on lui attribue. Quant à la chair, le narrateur est toujours aussi discret. Mais il faut ici encore faire appel au bon sens. Il n'y a d'huîtres vides qui ne furent pleines avant. Ces militaires ont mangé des huîtres. Pourquoi le héros n'aurait-il pas fait comme eux? Notons que l'esthétique et le gastronomique s'avèrent une fois de plus inextricablement liés: les plats servis par le chef "donnent autant de plaisir à l'imagination qu'à la gourmandise", écrit Proust. On imagine difficilement le héros faisant le dégoûté dans ces circonstances. L'ami de SaintLoup a fait comme les autres: il s'est pourléché, il a tout mangé sans laisser une miette.

Sans doute le contexte y est-il pour quelque chose. C'est la soirée de l'amitié, on s'apprécie, on s'aime. Ces jeunes gens sont entre eux, et ils s'en trouvent très bien. Ce qui provoque d'ailleurs chez l'un d'eux, l'ami en titre, une petite crise de jalousie:

"Je suis jaloux, je suis furieux", me dit Saint-Loup, moitié en riant, moitié sérieusement, faisant allusion aux interminables conversations à part que j'avais avec son ami. "Est-ce que vous le trouvez plus intelligent que moii? Est-ce que vous l'aimez mieux que moi? Alors, comme ça, il n'y en a plus que pour lui?" (CG, II, 4I7) 
Et Proust d'ajouter, pour qu'il n'y ait pas de malentendu:

(Les hommes qui aiment énormément une femme, qui vivent dans une société d'hommes à femmes se permettent des plaisanteries que d'autres qui y verraient moins d'innocence n'oseraient pas).

Honni soit qui mal y pense. Certes oui. Mais s'il n'y a vraiment rien de coupable ici, pourquoi nous parle-t-on de culpabilité? À l'évidence, l'assemblée est hétérosexuelle. L'espèce d'avertissement qu'on nous fait est donc sans raison d'être, nous ne voyons vraiment pas pourquoi il a fallu l'ajouter ici. À moins qu'il faille comprendre que le narrateur appartient lui-même à la catégorie d'hommes qu'il évoque, ceux "qui y voient moins d'innocence", qui ont des choses à se reprocher. En ce cas, ce passage serait aussi un aveu: volontaire ou involontaire, laïsons la question en suspens pour l'instant. "C'est celui qui le dit qui le fait", soupçonnent les enfants qui ont fort bien compris que n'importe qui ne peut pas énoncer n'importe quoi dans n'importe quel contexte donné. ${ }^{+}$ Serait-ce donc cette maxime fondatrice de l'herméneutique enfantine qu'il convient d'appliquer ici? En rappelant en même temps cette phrase de La Prisonnière, dans un autre commentaire sur Sodome, où le même soupçon est formulé:

L'objet de ses études, pour un psychiatre, réagit souvent sur lui. Mais avant cela, quelle obscure inclination, quel fascinateur effroi le lui avait fait choisir? ( $P, \mathrm{III}$, 7II)

Notons que le narrateur du Côté de Guermantes qualifie de "plaisanterie" l'intervention de son ami, alors que plus haut il a remarqué que Saint-Loup ne plaisante qu'à moitié: "moitié en riant, moitié sérieusement". Nous fronçons les sourcils. Nous savons que l'étiquette "hommes à femmes" ne convient que partiellement au fringant militaire, personnage fort secret, menant une double vie. Nous nous rappelons entre autres certaines révélations d'Aimé, le maître d'hôtel de Balbec, et qui portent justement sur l'époque où le héros proustien est en villégiature à la côte:

4. Sur ce principe, voir A. Compagnon: "La dernière victime du narrateur", Critique, 598, 1997. L'article de Compagnon est un excellent compte rendu du livre de M. Lavagetto, Chambre 43. Un lapsus de Marcel Proust, Belin, 1996. 
"Mais oui, monsieur, me dit-il, c'est archiconnu, il y a bien longtemps que je le sais." (TR, IV, 259)

Et plus loin, à propos du même (c'est le narrateur qui parle):

Je me rappelais aussi son affection pour moi, sa manière tendre, sentimentale de l'exprimer et je me disais que cela non plus, qui eût pu tromper quelque autre, signifiait alors tout autre chose, même tout le contraire, de ce que j'apprenais aujourd'hui. (TR, IV, 265).

Mais il ne faut pas juger trop vite en cette matière oh combien délicate. Ce qui importe ici, c'est le changement qu'on observe, la métamorphose qui a eu lieu. Les huîtres n'inspirent que de la répulsion au jeune héros quand il est à la mer avec Mme de Villeparisis et sa grand-mère. Mais ses goûts changent. Et quand il dîne à Doncières “entre hommes", c'en est fini de son aversion. L'ostréophobe s'est mué en ostréophile. Il est devenu un autre homme.

Dans la fameuse tirade contre l'homosexualité au début de Sodome et Gomorrhe, Proust évoque entre autres: "les cas où l'on verra que l'inversion est guérissable" (sG, III, 26). Ce “cas" n'est pas vraiment évoqué dans le texte, la digression à laquelle il est fait allusion ici n'ayant pas été reprise dans la version publiée du livre: il faut la lire dans la section des "Esquisses", accident génétique comme il y en a quelques autres dans les romans posthumes. En un sens, c'est dommage pour nous, car nous retrouvons le motif de l'huître dans ce fragment non retenu. Voici le texte de l' "Esquisse":

Chez certains, bien rares, le mal n'est pas congénital [...] et dans ce cas, superficiel, il peut guérir. Quelquefois même il tient à une difficulté de faire l'amour avec une femme qui tient à une infirmité anatomique, or on guérit certains asthmes en détruisant des adhérences que le malade a dans le nez; d'autres fois il a pour cause un dégoût des femmes, une répulsion causée par leur odeur, par la qualité de leur peau, répulsion qui peut être vaincue, comme certains enfants qui se trouvent mal en voyant des huîtres ou du fromage finissent par les aimer beaucoup [...] ("Esquisse IV", SG, III, 95I)

L'idée à retenir est évidemment qu'on peut surmonter l'homosexualité de la même manière que certains parviennent à vaincre une répulsion alimentaire. 
Apprendre à manger des huîtres ou devenir hétéro, en somme, cela se fait de la même façon, suggère Proust, le principe est pareil, le cheminement similaire.

L'huître a disparu de la version du roman où le narrateur évoque une catégorie d'inverti bien spécifique qu'il appelle le "solitaire". Puisque le "solitaire" évolue dans un paysage marin, nous songeons à Balbec:

Alors le solitaire languit seul. Il n'a d'autre plaisir que d'aller à la station de bains de mer voisine demander un renseignement à un certain employé de chemin de fer. Mais celui-ci a reçu de l'avancement [...] le solitaire ne pourra plus aller lui demander l'heure des trains [...] et avant de rentrer rêver dans sa tour, comme Grisélidis, il s'attarde sur la plage, telle une étrange Andromède qu'aucun Argonaute ne viendra délivrer, comme une méduse stérile qui périra sur le sable [...] (SG, III, 27-28)

La méduse était l'autre objet de dégoût dans la scène du déjeuner d'À l'ombre des jeunes filles en fleurs ("la viscosité des méduses [...] me ternissait la plage de Balbec"). Ce qui donne à croire qu'un principe de "traductibilité" existe entre ces deux termes: huître ou méduse, les deux signifiants sont proches, voire s'équivalent; à la limite, on peut dire l'un pour l'autre, cela ne change guère le sens du texte. Dans le passage qu'on vient de citer, Proust se trompe de mythe: c'est Persée, et non pas l'Argonaute, qui est venu au secours d'Andromède. ${ }^{5}$ Mais l' "argonaute" est aussi le nom d'un mollusque et il n'est pas interdit de penser que le romancier s'est peut-être délibérément servi de ce terme afin de ne pas quitter le registre gastronomique et ostréicole. Notons que Proust épistolier, isolé et malade, se compare volontiers à Andromède. Ce qu'il retient alors de l'allusion mythologique, c'est la figure de la captive "attachée à son rocher". ${ }^{6}$ Pour nous, ce "rocher" évoque l'huître. C'est un autre indice qu'une sorte de configuration thématique existe dans l'esprit de l'écrivain reliant l'huître, la méduse et le motif de l'homosexualité.

Dans le texte de l'"Esquisse", Proust ne dit pas quelle est la station de bains de mer où il fait rêver son "solitaire". Le narrateur de Sodome et Gomorrhe, quant à lui, se souvient de Balbec:

5. Persée est aussi le héros mythologique qui a tué la Méduse. Le nommer ici, c'eût été introduire une sorte d'inconséquence lexicale: le vainqueur de la "Méduse" ne peut être en même temps le secouriste d'une "méduse". Ce qui n'empêche pas qu'un rapport métaphorométonymique relie l'animal marin au monstre mythologique.

6. Cf. entre autres " $[. .$.$] une Andromède masculine toujours attachée à son rocher et qui souffre$ de voir Antoine Bibesco s'éloigner" (à Antoine Bibesco, juin 1902). Pour d'autres exemples, voir la note des éditeurs, SG, III, I285. 
Quand je ne suivais que mon instinct, la méduse me répugnait à Balbec; mais si je savais la regarder, comme Michelet, du point de vue de l'histoire naturelle et esthétique, je voyais une délicieuse girandole d'azur. (SG, III, 28)

C'est faire apparaître, de manière explicite, le souvenir du déjeuner avec Mme de Villeparisis. Et on comprend mieux à ce moment à quoi sert le motif de la méduse chez Proust: c'est le chaînon manquant entre l'huître et l'inversion, c'est la petite passerelle dont nous avons besoin pour voyager de l'une à l'autre. Certes, tous les mangeurs d'huîtres ne sont pas des invertis dans La Recherche et vice versa. Mais un lien existe entre les deux, et c'est ce lien qui compte pour nous ici.

Nous n'en avons pas fini avec les figures de la répulsion nutritive. Voici un autre élément qu'il convient de mentionner et qui vient enrichir notre liste. En lisant la tirade contre les homosexuels de Sodome et Gomorrhe, nous repensons également à une page $d u$ Côté de chez Swann, page assez célèbre du reste où le narrateur évoque les prouesses culinaires de Françoise. Il s'agit de l'évocation des asperges dont Jean-Pierre Richard a célébré "le phallisme tendre":?

[...] mais mon ravissement était devant les asperges, trempées d'outre-mer et de rose et dont l'épi, finement pignoché de mauve et d'azur, se dégrade insensiblement jusqu'au pied - encore souillé pourtant du sol de leur plant - par des irisations qui ne sont pas de la terre. (s, I, Ing)

Nous sommes à Combray, en terre ferme. Et pourtant, l'ambiance est on ne peut plus marine. Les commentateurs de ce passage n'ont en effet eu aucune difficulté à démontrer que Proust s'amuse ici à pasticher une page de La Merde Micheler, page où le grand historien évoque... la méduse: "La délicieuse créature, avec son innocence visible et l'iris de ses douces couleurs" ${ }^{\prime 8}$ On voit bien ici comment Proust quand il se met sur le chapitre de l'homosexualité ne cesse donc de superposer images et figures, déployant dans son texte un vaste réseau métaphorico-métonymique dont la fonction est aussi de remettre sans cesse à plus tard la nomination du sens "propre": le plaisir de l'écrivain est dans la

7. Proust et le monde sensible, Seuil, ("Points"), 1974, p. 133.

8. Michelet, La Mer, éd. présentée, établie et annotée par Jean Borie, Gallimard, "Folio", p. 152 . 
multiplication des images; identifier l'objet, ce serait mettre fin au plaisir; c'est pourquoi l'objet n'est jamais "proprement" nommé.

Notons que nous retrouvons dans la scène des asperges le motif du "dégoût" qui est lié à Balbec et à l'épisode du Grand Hôtel. Mais un phénomène de déplacement peut être observé; le romancier a en quelque sorte "dévié" ce motif vers un autre personnage: le narrateur est "ravi” par ces belles asperges qu'on lui sert, mais à la fille de cuisine, qui doit les éplucher, leur odeur donne des crises d'asthme." Et nous nous rappelons aussi, dans un autre registre, ces paroles du duc de Guermantes évoquant un tableau d'Elstir où les mêmes tiges comestibles sont à l'honneur:

Il n'y avait que cela dans le tableau, une botte d'asperges précisément semblables à celles que vous êtes en train d'avaler. Mais moi, je me suis refusé à avaler les asperges de M. Elstir. (CG, II, 79I)

Comment ne pas penser au jeune homme de Balbec déjeunant avec sa grandmère? Le duc est littéralement horrifié par les asperges du peintre (on note le

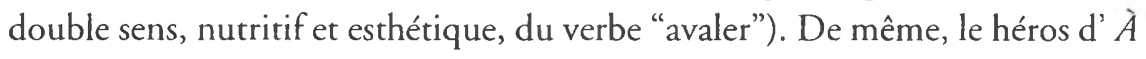
l'ombre des jeunes filles en fleurs déteste à la fois la chair des huîtres et celle des méduses. Mais le duc n'est qu'un rustre bouché et insensible qui n'évolue pas, alors que le héros du roman parviendra à surmonter son ostréophobie, et qu'il finira par aimer les "mauves orchidées de la mer" (sG, III, 28). C'est que le héros a une sensibilité d'artiste, c'est que l'art lui permet d'aller au-delà de son dégoût.

Le moment est venu de procéder à une récapitulation. Trois séries, semble-t-il, s'entrecroisent dans l'oeuvre:

- une série "gustative"

- une série "esthétique"

- une série "sexuelle".

Dans les trois cas, la série est évoquée dans un contexte d'apprentissage et de Bildung. Ces groupements que nous dégageons participent du mouvement ascendant du roman qui raconte, comme on sait, l'histoire d'une vocation: un jeune homme maladif et sans volonté se sent appelé à devenir un très grand 
écrivain. Le texte retrace ce parcours sans aller jusqu'à la fin: le livre s'arrête au moment où le personnage commence à écrire. À leur façon, nos trois séries préparent et accompagnent cette aventure. À travers elles, nous voyons comment un être initialement borné, enfermé dans ses manies et ses phobies, parvient à dépasser ce cadre initial, pour aller vers un "plus":

- un "plus" de plaisir gastronomique dans la série "gustative"

- un "plus" d'émotion esthétique dans la série "esthétique"

- une meilleure santé dans la série "sexuelle" (cf. les pages que nous avons lues de Sodome et Gomorrhe).

Mais voici quel est le problème qui se présente à nous. Si la troisième série fait partie de l'ensemble, nous ne voyons pas tout de suite en quoi elle concernerait le héros du roman. Le jeune déjeuneur de Balbec n'est pas un inverti mais un hétéro actif et "dragueur". S'il est confronté à la double tâche de cultiver son palais et d'éduquer son regard, sexuellement parlant, son éducation est faite, il n'a plus rien à apprendre. Certes, sa vie affective est loin d'être heureuse, et sa liaison avec Albertine finit en catastrophe. Il n'en reste pas moins que son "orientation" est saine: ce jeune homme est d'emblée du "bon" côté.

Mais l'est-il vraiment? Là est très exactement la difficulté qui se présente à nous. En réfléchissant un peu plus profondément à ce que nous dit le texte, nous nous rendons compte que nous ne savons finalement pas très bien quel est le bon "côté" en l'occurrence. Qu'est vraiment l'inversion du point de vue du narrateur proustien? Est-ce un "moins" ou un "plus"? Chez le héros du livre, l'hétérosexualité est au départ, comme se situent au départ sa phobie huîtrière et son "incapacité de savoir regarder" (TR, IV, 234). Or si l'ensemble évolue, en bonne logique, la sexualité devra évoluer dans le même sens. Qui plus est, dans un roman d'apprentissage, on évolue nécessairement d'un "moins" vers un "plus", c'est la loi du genre. L'homosexualité, serait-elle donc un "plus" chez Proust? Serait-ce donc là ce qu'à travers ces dérives et détours on essaie de nous dire?

Dis-moi ce que tu manges et je te dirai comment tu aimes, adage fondateur de la gastronomie proustienne. ${ }^{10}$ Il s'ensuit que si un personnage apprend à se

I0. La très gomorrhéenne Albertine apprécie beaucoup les huîtres. Dans la scène des "nourritures criées", elle désire d'ailleurs successivement tous les produits dont elle entend crier le nom par la marchande de poissons (P, III, 633)... Pour une interprétation "hétérosexuelle" de cette question, cf. L. Dallenbach, "À l'origine de La Recherche ou la 'raie du jour"” dans Proust contemporain, ét. réunies par $\mathrm{S}$. Bertho, $C R I N \mathrm{n}^{\circ} 28,1994$. Mais ce que nous avons voulu montrer 
nourrir autrement, cela devrait logiquement entraîner des changements dans son comportement amoureux. Si on applique ce principe au héros du roman, force est d'admettre que nous évoluons vers une homosexualité acceptable, voire souhaitable, alors même que celle-ci est sévèrement condamnée dans le texte.

Certes, le scénario que nous imaginons reste à l'état hypothétique; Proust ne raconte pas dans son livre le devenir homosexuel de son héros. Mais peu importe, ajouterions-nous. Si nous ne voyons pas Marcel passer du côté de Sodome, nous ne le voyons pas non plus devenir un grand écrivain. Dans les deux cas, le moment d'aboutissement est situé hors roman. C'est le point de fuite du texte, le passage à l'autre côté du miroir; la chose troublante étant évidemment que c'est dans un même lieu que l'écrivain localise l'inversion et la vocation littéraire.

Gardons-nous, une fois de plus, de conclure trop hâtivement. C'est d'abord à un paradoxe que Proust essaie de nous rendre sensibles: pas de goût sans dégoût, le dégoût fait l'envie, les psychanalystes le savent bien, l'auteur de $L a$ Recherche nous l'explique à sa manière. Songeons à Swann qui se dit physiquement "dégoûté" par Odette dont il deviendra pourtant follement amoureux (s, I, 193). Ou au petit groupe des "fidèles" voyageant avec l'amant de Charlie dans le petit "tortillard" qui les amène à la Raspelière. Le baron exerce une indiscutable fascination sur eux, son étrangeté attire. C'est que la répulsion est un facteur d'attraction:

Mais au fond, si M. de Charlus ne venait pas, on était presque déçu de voyager seulement entre gens comme tout le monde et de n'avoir pas auprès de soi ce personnage peinturluré, pansu et clos, semblable à quelque boîte de provenance exotique et suspecte qui laisse échapper la curieuse odeur de fruits auxquels l'idée de goûter seulement vous soulèverait le coeur. (sG, III, 429)

"Rien que l'idée vous soulèverait le coeur". C'est que l'idée est bien là et que la frontière est bien mince chez Proust entre détestation et désir. Mais ce n'est là qu'un premier temps du raisonnement et il faut aller plus loin. Car on se rend compte une fois de plus ici que le paradoxe que formule le texte ne laisse pas inentamé le parcours du héros. Charlus, il est vrai, est un bonhomme vieillissant, sa beauté est passée. Mais imaginons le même épisode avec un être plus jeune et qui ferait l'objet d'un même type d'attention. C'est ce que Proust fait dans l'avant-texte où il revient à la figure du "solitaire" se promenant au bord de l'eau:

ici, c'est que la seule clé hétérosexuelle ne suffit pas. 
J'eus un vrai plaisir un jour à voir qu'un gros temps avait déposé sur le sable d'innombrables méduses. Je m'enchantais à voir le soleil briller dans les lustres d'opale, même je touchai leur délicate ceinture lilas avec autant de joie que si ç'avait été l'écharpe d'Iris. De dégoût je n'en avais aucun car le sentiment esthétique nous fait franchir les limites qu'imposent à nos goûts les préférences du corps. C'est ainsi qu'un grand artiste pourra comparer à de belles Muses, pourra s'enchanter à regarder de jeunes hommes que trouverait écoeurants un homme de club, livré aux étroites répulsions de l'instinct sexuel. (JF, II, var. a de la p. 306,1488 )

Le même texte apparaît une seconde fois avec quelques minimes changements (Proust travaille ainsi, accumulant plusieurs versions successives d'une même page):

J'eus un vrai plaisir un jour à voir qu'un gros temps avait déposé sur le sable d'innombrables méduses. Je m'enchantais, à voir le soleil briller dans leurs lustres d'opale, même je touchai leur délicate ceinture lilas avec autant de joie que si ç'avait été l'écharpe d'Iris. De dégoût je n'en sentis aucun, car le sentiment esthétique nous fait franchir les limites qu'imposent à nos goûts les préférences du corps. C'est ainsi qu'un grand artiste pourra comparer à de belles Muses, pourra s'enchanter à regarder de jeunes hommes que trouverait écoeurants un homme de club, livré à une étroite répulsion de l'instinct sexuel. (ibid., I489)

Chaque mot a son importance: la "muse" est liée à la "méduse", phonétiquement mais aussi du point de vue du référent (le "clubman" est un peu, par rapport à ces "hommes jeunes" ce que l'Argonaute est à la méduse dans le passage de Sodome et Gomorrhe que nous avons commenté); la "ceinture" qui est "touchée" est une métaphore de la rencontre sexuelle (Bloch à propos d'Odette: “Je l'avais rencontrée quelques jours auparavant dans le train de Ceinture. Elle voulut bien dénouer la sienne en faveur de ton serviteur", JF, II, 136); le motif de l' "irisation" nous renvoie à la description des asperges de Combray, les "lustres d'opale" nous rappelant par ailleurs les "gouttes d'eau lustrale" dans le passage du déjeuner du Grand Hôtel.

Mais dans le contexte de notre enquête, l'idée à retenir est surtout que l'art, s'il est vrai, s'il est "grand", n'est pas sexuellement correct chez Proust, c'est-àdire que l'expérience esthétique fait éclater les cadres d'une sexualité dite "normale". Partant, que celui qui cherche à vaincre une répulsion sexuelle n'a pas à s'adresser au clinicien, seul l'artiste pourra l'aider. Proust n'est donc pas 
loin de suggérer que le héros de son livre doit apprendre à aller contre son instinct sexuel, comme il devra aussi aller vers l'art.

Ultime averrissement à ceux qui s'obstineraient à mal nous comprendre: l'aveu “oui, je suis homosexuel" n'apparaît pas chez Proust, quoi qu'on en ait pu dire, quels qu'aient pu être les rêves de certains. ${ }^{\text {II }}$ On s'y attend pourtant, là est la difficulté. Comme l'a bien montré Antoine Compagnon, nous sommes, de ce point de vue, condamnés à "une perplexité interminable". ${ }^{12}$ Il y a à notre "perplexité" bien des raisons: narratologiques (écrivain et narrateur se ressemblent et pourtant ne coïncident pas), génétiques (une censure apparaît parfois, on l'a constaté ici même, quand on passe del'avant-texte au texte), stylistiques: Proust est romancier, non pas sexologue; l'homosexualité est un phénomène qu'il cherche à décrire, non, sans doute, à expliquer. ${ }^{13}$ Il faut en tirer les conséquences: l'affaire de l'écrivain est d'écrire, de faire en sorte que son écriture avance. La question importante n'est donc pas de ce point de vue ce que cela veut dire mais bien: comment cela est-il dit? C'est ce que nous avons voulu illustrer ici.

II. P. Sollers ironise à ce sujet: "On peut imaginer le sourire de Proust [...] devant les paquets qui lui arrivent chaque jour d'Amérique contenant des dizaines de livres de Gay and Lesbian Studies" (L'oeil de Proust. Les dessins de Marcel Proust, Stock, 1999, p. 30).

I2. Art. cité, p. I42.

13. Lawrence R. Schehr a trouvé une formule aussi compacte qu'efficace: "Homosexuality is an excuse for discourse" ("Interpreting Proustian Interpretation", The Shock of Men. Homosexual Hermeneutics in French Writing, Stanford University Press, 1995, p. 71). 